\title{
A mesh-free finite point method for advective-diffusive transport and fluid flow problems
}

\author{
E. Oñate, S. Idelsohn
}

Abstract The finite point method (FPM) is a gridless numerical procedure based on the combination of weighted least square interpolations on a cloud of points with point collocation for evaluating the approximation integrals. In the paper, details of a procedure for stabilizing the numerical solution for advective-diffusive transport and fluid flow problems using the FPM are given. The method is based on a consistent introduction of the stabilizing terms in the governing differential equations. One example showing the applicability of the FPM is given.

\section{1}

\section{Introduction}

A number of authors have recently investigated the possibility of deriving numerical methods where meshes are unnecessary. The different methods can be classified as Finite Difference (FD) schemes (Forsythe and Wasow 1960; Perrone and Kao 1975; Liszka and Orkisz 1980; Liszka 1984), Smooth Particle Hydrodynamic (SPH) methods (Moraghan 1988), Diffuse Element (DE) method (Nayroles et al. 1992), Element Free Galerkin (EFG) method (Belytschko et al. 1994 1995; Taylor et al. 1995; Belytschko 1998), Reproducing Kernel Particle (RPK) method (Liu et al. 1995a,b; Liu et al. 1996a,b; Liu et al. 1997), Partition of Unity (PU) based methods (Duarte and Oden 1995; Baduška and Melenk 1995) and Finite Point (FP) method (Oñate et al. 1995a,b; Oñate et al. 1996a,b, Fischer et al. 1995; Fischer 1996). The basic ingredient of all these methods is the existance of an arbitrary collection of points. The points are grouped together in "clouds" where a local interpolation for the problems variables is written. DE, EFG, RPK and PU methods typically use a background grid for computing numerically the integrals

Communicated by D. E. Beskos, 15 November 1997

\section{E. Oñate}

International Center for Numerical Methods in Engineering (CIMNE) Gran Capitán, s/n, E-08034 Barcelona, Spain

S. Idelsohn

Universidad Nacional del Litoral, Santa Fe,

Argentina Visiting Professor, CIMNE

Correspondence to: E. Oñate

The authors are grateful to Dr. T. Fischer for providing results for the example analyzed. resulting from weighted residual forms of the governing differential equations. On the other hand, FD, SPH and FP methods are truly gridless or mesh-free procedures as the set of algebraic equations is derived via simpler point collocation procedures. A detailed comparison of all these methods can be found in (Oñate et al. 1995).

The work of the authors has been recently focussed in the development of the so called Finite Point Method (FPM). The simpler FPM is based on combining a weighted least square interpolation within each cloud where a weighting function is kept "fixed", with a point collocation scheme to derive the approximation equations. The efficiency of the FPM for solving a range of problems has been reported by Oñate et al. (1995a,b, 1996a,b), Fischer et al. (1995) and Fischer (1996).

In a recent paper (Oñate et al. 1996b) the authors have shown that the successful use of the FPM for solving advective-diffusive transport and fluid flow problems relies on the adequate stabilization of the numerical solution.

Here, the standard SUPG, GLS, Taylor-Galerkin, etc. procedures used in FE and FV methods are just not applicable, as the integral form of the equations is not available. In Oñate et al. (1996b) the authors proposed to use an "ad hoc" upwind stabilizing scheme similar to that used in finite difference methods. A new stabilization technique proposed by Oñate $(1996,1998)$ based on the consistent modification of the governing differential equations originally was successfully applied to a range of advectivediffusive and fluid flow problems using the FPM (Onate et al. 1996b).

The layout of the paper is as follows. In next section the basis of the FPM are briefly described. Then, the principles of the new stabilization technique for advective-diffusive problems are presented and the corresponding FPM equations are derived for both steady state and transient cases. The concepts are then extended to the solution of compressible and incompressible fluid mechanics equations via the FPM. The paper ends up with one example showing the efficiency and applicability of the stabilized FPM for solving fluid flow problems.

\section{2}

\section{Basic concept of the FPM}

Let us assume a scalar problem governed by a differential equation

$A(u)=b \quad$ in $\quad \Omega$

with Neumann boundary conditions

$B(u)=t \quad$ in $\quad \Gamma_{t}$ 
and Dirichlet (essential) boundary conditions

$$
u-u_{p}=0 \quad \text { in } \quad \Gamma_{u}
$$

to be satisfied in a domain $\Omega$ with boundary $\Gamma=\Gamma_{t} \cup \Gamma_{u}$. In the above $A$ and $B$ are appropriate differential operators, $u$ is the problem unknown and $b$ and $t$ represent external forces or sources acting over the domain $\Omega$ and along the boundary $\Gamma_{t}$, respectively. Finally $u_{p}$ is the prescribed value of $u$ over the boundary $\Gamma_{u}$.

The most general procedure of solving numerically the above system of differential equations is the weighted residual method in which the unknown function $u$ is approximated by some trial approximation $\hat{u}$ and Eqs. (1) and (2) are replaced by (Zienkiewicz and Taylor 1991)

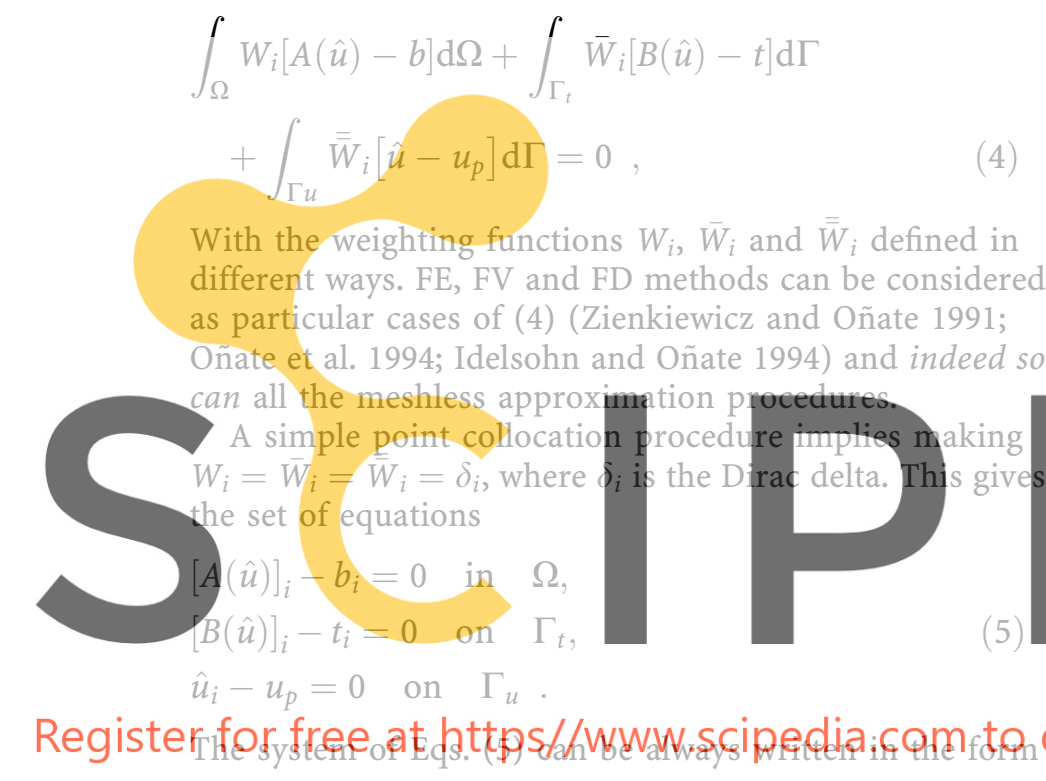

$\mathrm{Ka}=\mathrm{f}$

where $\mathbf{K}$ is the coefficient matrix which symmetry is not generally achieved in point collocation procedures, vector a contains the problem unknowns and $\mathbf{f}$ is a vector containing contributions from the external sources and forces and the prescribed values $u_{p}$.

In order to keep a local character of the problem (leading to a banded matrix), function $u$ must be approximated by a combination of locally defined functions as

$u(x) \cong \hat{u}(x)=\sum_{i=1}^{n_{p}} N_{i}(x) u_{i}^{h}=\mathbf{N}^{\mathrm{T}}(x) \mathbf{u}^{h}$,

with $n_{p}$ being the total number of points in the domain and the interpolation functions $N_{i}(x)$ satisfy

$\begin{array}{lll}N_{i}(x) \neq 0 & \text { if } & x \in \Omega_{i}, \\ N_{i}(x)=0 & \text { if } & x \notin \Omega_{i} .\end{array}$

Here $\Omega_{i}$ is a subdomain of $\Omega$ containing $n$ points, $n \ll n_{p}$. In (7) $u_{i}^{h}$ is the approximate value of $u$ at point $i$ such that $u\left(x_{i}\right) \simeq u_{i}^{h}$.

The way to define the shape functions $N_{i}$ is classical in the FEM (Zienkiewicz and Taylor 1991). The derivation of $N_{i}$ in the FPM will be described next. More details can be found in Oñate et al. (1996a,b).
3

\section{Derivation of interpolation function via fixed weighted least square approximations}

Let $\Omega_{i}$ be the interpolation domain (cloud) of a function $u(x)$ and let $s_{j}$ with $j=1,2, \ldots, n$ be a collection of $n$ points with coordinates $x_{j} \in \Omega_{i}$. The unknown function $u$ may be approximated within $\Omega_{i}$ by

$u(x) \cong \hat{u}(x)=\sum_{l=1}^{m} p_{l}(x) \alpha_{l}=\mathbf{p}(x)^{\mathrm{T}} \boldsymbol{\alpha}$,

where $\boldsymbol{\alpha}=\left[\alpha_{1}, \alpha_{2}, \ldots, \alpha_{m}\right]^{\mathrm{T}}$ and vector $\mathbf{p}(x)$ contains typically monomials, hereafter termed "base interpolating functions", in the space coordinates ensuring that the basis is complete. For a 2D problem we can specify

$\mathrm{p}=[1, x, y]^{\mathrm{T}}$ for $m=3$

and

$\mathrm{p}=\left[1, x, y, x^{2}, x y, y^{2}\right]^{\mathrm{T}}$ for $m=6$ etc.

Function $u(x)$ can now be sampled at the $n$ points belonging to $\Omega_{i}$ giving

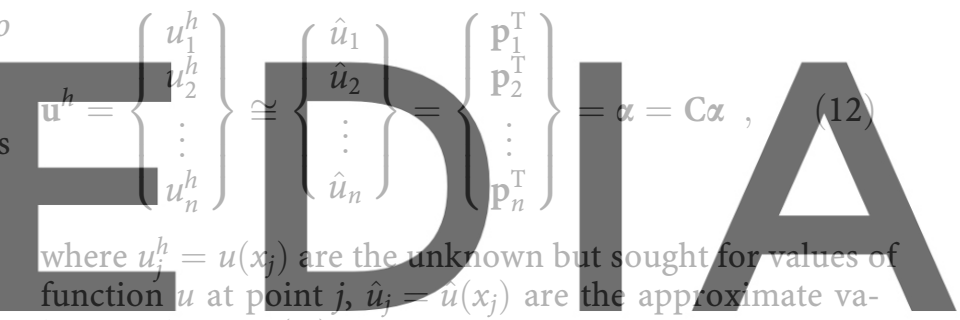

lues, and $\mathrm{p}_{j}=\mathrm{p}\left(x_{j}\right)$.

In the FE approximation the number of points is chosen

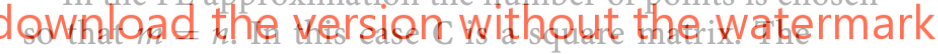
procedure leads to the standard shape functions in the FEM (Zienkiewicz and Taylor 1991).

If $n>m, \mathbf{C}$ is no longer a square matrix and the approximation can not fit all the $u_{j}^{h}$ values. This problem can be simply overcome by determining the $\hat{u}$ values by minimizing the sum of the square distances of the error at each point weighted with a function $\varphi(x)$ as

$$
\begin{aligned}
J & =\sum_{j=1}^{n} \varphi\left(x_{j}\right)\left(u_{j}^{h}-\hat{u}\left(x_{j}\right)\right)^{2} \\
& =\sum_{j=1}^{n} \varphi\left(x_{j}\right)\left(u_{j}^{h}-\mathbf{p}_{j}^{\mathrm{T}} \boldsymbol{\alpha}\right)^{2}
\end{aligned}
$$

with respect to the $\alpha$ parameters. Note that for $\varphi(x)=1$ the standard least square (LSQ) method is reproduced.

Function $\varphi(x)$ is usually built in such a way that it takes a unit value in the vicinity of the point $i$ typically called "star node" (Liszka 1984) where the function (or its derivatives) are to be computed and vanishes outside a region $\Omega_{i}$ surrounding the point. The region $\Omega_{i}$ can be used to define the number of sampling points $n$ in the interpolation region. A typical choice for $\varphi(x)$ is the normalized Gaussian function. Of course $n \geq m$ is always required in the sampling region and if equality occurs no effect of weighting is present and the interpolation is the same as in the LSQ scheme (Batina 1993). 
Standard minimization of Eq. (13) with respect to $\alpha$ gives

$\boldsymbol{\alpha}=\overline{\mathbf{C}}^{-1} \mathbf{u}^{h}, \quad \overline{\mathbf{C}}^{-1}=\mathbf{A}^{-1} \mathbf{B}$.

The final approximation is obtained substituting the value of $\alpha$ in Eq. (14) into (9) giving

$\mathbf{A}=\sum_{j=1}^{n} \varphi\left(x_{j}\right) \mathbf{p}\left(x_{j}\right) \mathbf{p}^{\mathrm{T}}\left(x_{j}\right)$

$\mathbf{B}=\left[\varphi\left(x_{1}\right) \mathbf{p}\left(x_{1}\right), \varphi\left(x_{2}\right) \mathbf{p}\left(x_{2}\right), \ldots, \varphi\left(x_{n}\right) \mathbf{p}\left(x_{n}\right)\right]$.

The final approximation is obtained by substituting Eqs. (14) into (9) giving

$\hat{u}(x)=\mathbf{p}^{\mathrm{T}} \overline{\mathbf{C}}^{-1} \mathbf{u}^{h}=\mathbf{N}^{\mathrm{T}} \mathbf{u}^{h}=\sum_{j=1}^{n} N_{j}^{i} u_{j}^{h}$,

where the "shape functions" are

$N_{j}^{i}(x)=\sum_{l=1}^{m} p_{l}(x) \bar{C}_{l j}^{-1}=\mathbf{p}^{\mathrm{T}}(x) \overline{\mathbf{C}}^{-1}$

It must be noted that accordingly to the least square character of the approximation

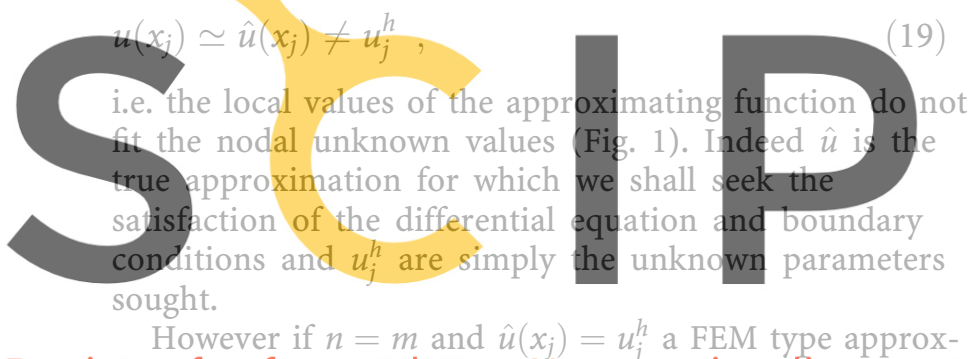

However if $n=m$ and $\hat{u}\left(x_{i}\right)=u_{i}^{h}$ a FEM type approx-

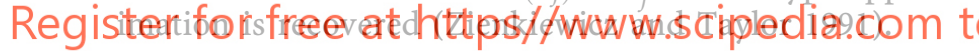

3.1

\section{Fixed least square approximation (FLS)}

The weighted least square approximation described above depends on a great extent on the shape and the way to apply the weighting function. The simplest way is to define a fixed function $\varphi(x)$ for each of the $\Omega_{i}$ interpolation domains (see Fig. 1).

Let $\varphi_{i}(x)$ be a weighting functions satisfying

$\varphi_{i}\left(x_{i}\right)=1$

$\varphi_{i}(x) \neq 0, x \in \Omega_{i}$;

$\varphi_{i}(x)=0 \quad x \notin \Omega_{i}$.

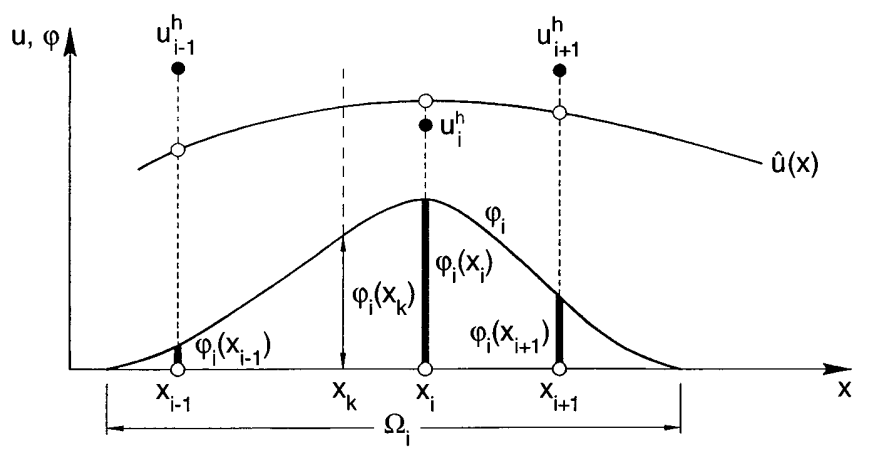

Fig. 1. Fixed weighing least square procedure
Then the minimization square distance becomes

$J=\sum_{j=1}^{n} \varphi_{i}\left(x_{j}\right)\left(u_{j}^{h}-\hat{u}\left(x_{j}\right)\right)^{2} \quad$ minimum .

The expression of matrices $\mathbf{A}$ and $\mathbf{B}$ are now

$\mathbf{A}=\sum_{j=1}^{n} \varphi_{i}\left(x_{j}\right) \mathbf{p}\left(x_{j}\right) \mathbf{p}^{\mathrm{T}}\left(x_{j}\right)$,

$\mathbf{B}=\left[\varphi_{i}\left(x_{1}\right) \mathbf{p}\left(x_{1}\right), \varphi_{i}\left(x_{2}\right) \mathbf{p}\left(x_{2}\right), \ldots, \varphi_{i}\left(x_{n}\right) \mathbf{p}\left(x_{n}\right)\right]$.

Note that according to (9), the approximate function $\hat{u}(x)$ is defined in each interpolation domain $\Omega_{i}$. In fact, different interpolation domains can yield different shape functions $N_{j}^{i}$. As a consequence a point belonging to two or more overlapping interpolation domains has different values of the shape functions which means that $N_{j}^{i} \neq N_{j}^{k}$. The interpolation is now multivalued within $\Omega_{i}$ and, therefore, for any useful approximation a decision must be taken limiting the choice to a single value (Oñate et al. 1996a,b). Indeed, the approximate function $\hat{u}(x)$ will be typically used to provide the values of the unknown function $u(x)$ and its derivatives in only specific regions within each interpolation domain. For instance by using point collocation we

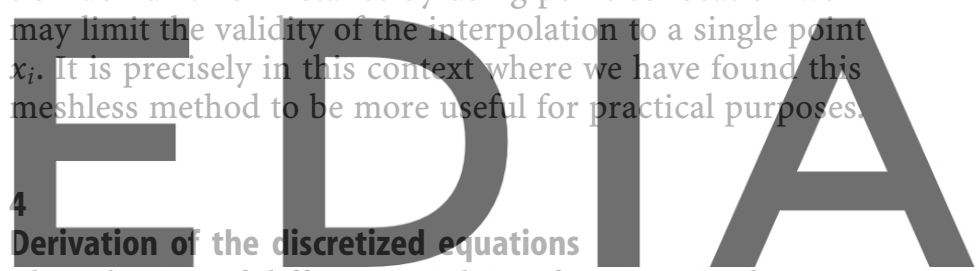

The selection of different weighting functions in the general weighted residual form of Eq. (3) yields different sets

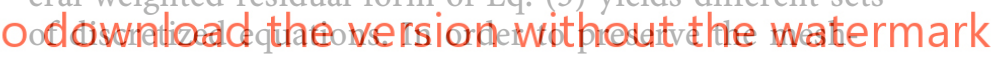

free character of the method, the weighting domain must be defined independently of any mesh. All approximation methods of integral type (i.e. Galerkin, area collocation, etc.) necessitate the introduction of complex procedures for integration, i.e. background grid, etc. (Nayroles et al. 1992; Belytschko et al. 1994; Lu et al. 1994; Liu et al.

1995a,b; Liu and Chen 1995; Liu et al. 1996). Some of these procedures are reviewed in Oñate et al. (1995a, 1996a,b). In this paper we shall limit the choice to point collocation methods where we feel the advantages of meshless procedures are best realized.

Point collocation implies making $W_{i}=\bar{W}_{i}=\overline{\bar{W}}_{i}=\delta_{i}$ in Eq. (3) where $\delta_{i}$ is the Dirac delta. This gives the set of equations

$$
\begin{aligned}
{[A(\hat{u})]_{i}-b_{i}=0 } & \text { in } \Omega, \\
{[B(\hat{u})]_{i}-t_{i}=0 } & \text { in } \Gamma_{t}, \\
\hat{u}_{i}-u_{p}=0 & \text { in } \Gamma_{u} .
\end{aligned}
$$

Any of the previous shape functions may be used now to approximate $\hat{u}$ leading in all cases to the system of equations

$\mathbf{K u}^{h}=\mathbf{f}$,

with $\left.K_{i j}=\left[A\left(N_{j}\right)\right]_{i}+B\left(N_{j}\right)\right]_{i}$ and where the symmetry of the "coefficient" matrix $\mathbf{K}$ is not generally achieved. Vector $\mathbf{u}^{h}$ contains the problem unknowns, $u_{i}^{h}$, and $\mathbf{f}$ is a vector 
containing the contributions from the force terms $b$ and $t$ and the prescribed values $u_{p}$.

\section{5}

\section{Application of the FPM to advective-diffusive transport problems}

Let us consider next the application of the FPM to the standard advection-diffusion equation given by

$$
\begin{aligned}
& \begin{aligned}
A(\phi) & =c \frac{\partial \phi}{\partial t}+\mathbf{u}^{\mathrm{T}} \nabla \phi-\nabla^{\mathrm{T}}(k \boldsymbol{\nabla} \phi)-Q \\
& =0 \text { in } \Omega, \\
B(\phi) & =\mathbf{n}^{\mathrm{T}} k \boldsymbol{\nabla} \phi+\bar{q}_{n}=0 \text { in } \Gamma_{q}, \\
\phi-\phi_{p} & =0 \text { in } \Gamma_{\phi},
\end{aligned}
\end{aligned}
$$

with the initial condition $\phi=\phi_{0}(x)$ for $t=t_{0}$.

In (26) $\nabla$ is the gradient operator, $c$ and $k$ are known physical parameters, $\mathbf{u}$ is the known velocity vector, $\phi$ the unknown field and $Q$ a source term. $\bar{q}_{n}$ and $\phi_{p}$ are known values of the flux and the known function at the boundaries $\Gamma_{q}$ and $\Gamma_{\phi}$, respectively.

It is widely accepted that the accurate numerical solution of advective-diffusive transport and fluid flow pro-

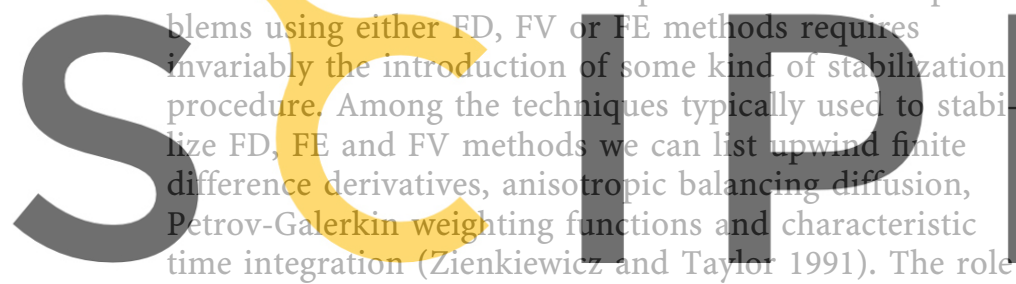
of this apparently non-physical stabilization is typically

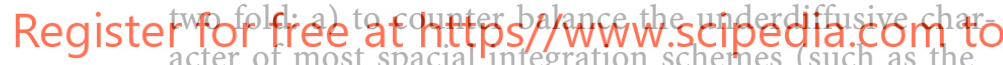
central difference scheme in FD or FV methods, or the equivalent Galerkin form in FEM, etc.), and b) to stabilize the numerical solution in the vecinity of high gradients (such as wave shocks etc.) by smoothing out local oscillations.

Although extensive work has been devoted to the development of stabilization procedures in $\mathrm{FD}, \mathrm{FV}$ and $\mathrm{FE}$ methods, it is acknowledged that most of the existing procedures are based on somewhat heuristic arguments. Indeed it is widely accepted that the origins and precise definition of the stabilization parameters used in numerical computations are in most cases unsolved mysteries, whose solution has motivated much research in recent years mainly within the FEM context (Zienkiewicz and Taylor 1991).

In a quite recent work Oñate $(1996,1998)$ has shown that the stabilization terms emerge naturally in the governing differential equations of the problem, once the concept of flow balance (or equilibrium) over a "finite" domain is accepted. This allows to reinterprete the stabilization terms as an intrinsic and natural contribution to the original differential equations, instead of an "extrinsic" correction term introduced at the discretization level, as it is usually understood by most FD, FV and FE practitioners. Moreover, by exploiting this natural stabilization concept, the standard forms of the balancing terms appearing in many well known stabilized schemes for ad- vective-diffusive and fluid flow problems such as direct artificial diffusion, upwinding and Petrov-Galerkin weighting, Streamline Upwind Petrov-Galerkin (SUPG), Subgrid Scale (SS), Galerkin Least Squares (GLS), LaxWendroff, Characteristic Galerkin, Laplacian pressure operator etc. are easily recognized and can be reinterpreted in a more physical manner. An additional important benefit from the approach proposed is that it provides an expression for computing numerically the stabilization parameter (Oñate 1996, 1998, Oñate et al 1997).

\section{1}

Stabilized form of the governing equation for advective- diffusive transport

The key to the successful development of a stabilized numerical FP scheme for analysis of advective type problems is the derivation of an "intrinsic" stabilized form of the differential equations governing the balance of fluxes in a finite domain.

For this purpose let us consider a finite rectangular domain of dimensions $h_{x}$ and $h_{y}$ in directions $x$ and $y$, respectively. Both the advective and diffusive fluxes are assumed to vary linearly along the four sides (Fig. 2). The flux balance equation will be obtained using the following

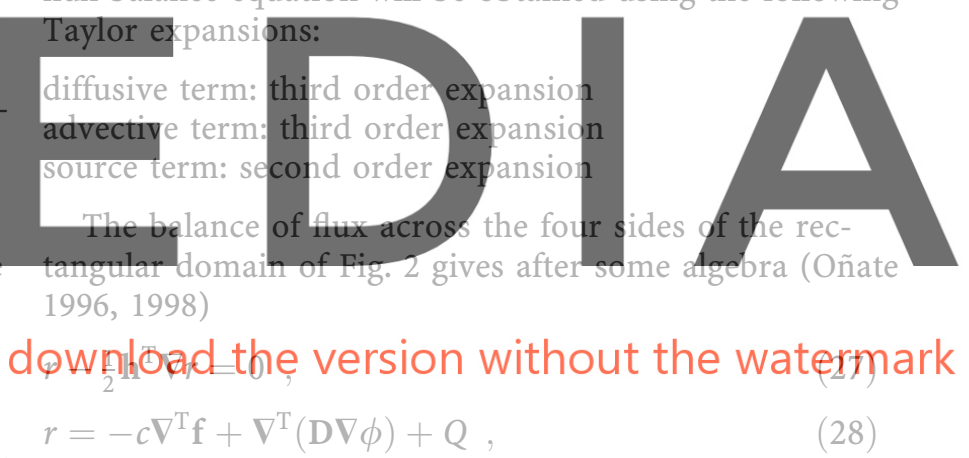

where

$\mathbf{f}=[u \phi, v \phi]^{\mathrm{T}}$,

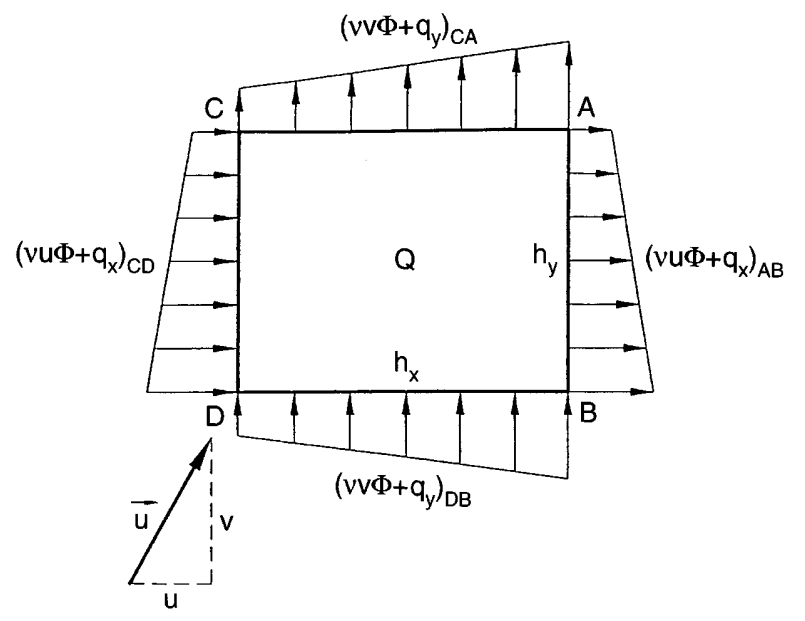

Fig. 2. Balance domain for $2 \mathrm{D}$ advection-diffusion problem. Advective and diffusive fluxes are assumed to vary linearly along the sides 
$\mathbf{D}=\left[\begin{array}{cc}k_{x} & 0 \\ 0 & k_{y}\end{array}\right]$

is the conductivity matrix

$\boldsymbol{\nabla}=\left[\frac{\partial}{\partial x}, \frac{\partial}{\partial y}\right]^{\mathrm{T}} \quad$ and $\quad \mathbf{h}=\left[h_{x}, h_{y}\right]^{\mathrm{T}}$.

Vector $\mathbf{h}$ is called the "characteristic length vector" and $h=|\mathbf{h}|$ is known as "characteristic length". Usually

$h=\alpha l^{(e)}$, where $\alpha$ is the sought stabilization parameter and

$l^{(e)}$ is an adequate element dimension (i.e. the element

length in 1D problems) (Oñate 1996).

The boundary conditions are written as

$\phi-\bar{\phi}=0 \quad$ on $\Gamma_{\phi}$,

where $\Gamma_{\phi}$ is the Dirichlet boundary, where the variable is prescribed, and

$\mathbf{n}^{\mathrm{T}} \mathbf{D} \boldsymbol{\nabla} \phi+\bar{q}_{n}-\frac{1}{2} \mathbf{h}^{\mathrm{T}} \mathrm{nr}=0 \quad$ on $\Gamma_{q}$ (33)

where $\bar{q}_{n}$ is the prescribed diffusive flux across the Neumann boundary $\Gamma_{q}$ with $\Gamma=\Gamma_{\phi} \cup \Gamma_{q}$ and $\mathbf{n}=\left[n_{x}, n_{y}\right]^{\mathrm{T}}$ is the normal vector.

Equation (33) has been obtained by balance of fluxes in the boundary domain of Fig. 3 us proximation for the advective and derivation can be found in Oña

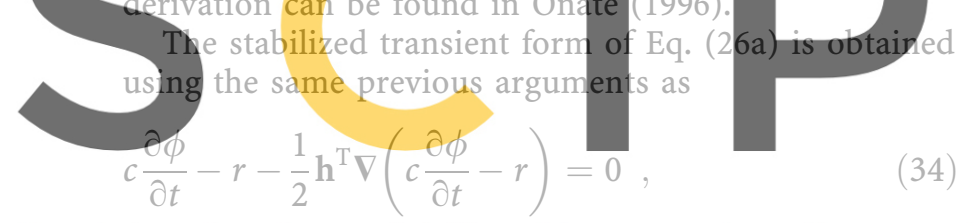

Register for free at https//www scipedia.com to

Note that the standard differential equations (26) are simply obtained neglecting the stabilizing terms in Eqs. (27)-(34) (i.e making $\mathbf{h}=0$ ).

\section{2}

\section{The concept of intrinsic time}

It can be further assumed that $h_{x}=h \cos \alpha$ and $h_{y}=$ $h \sin \alpha$, where $\alpha$ is the angle which the velocity vector

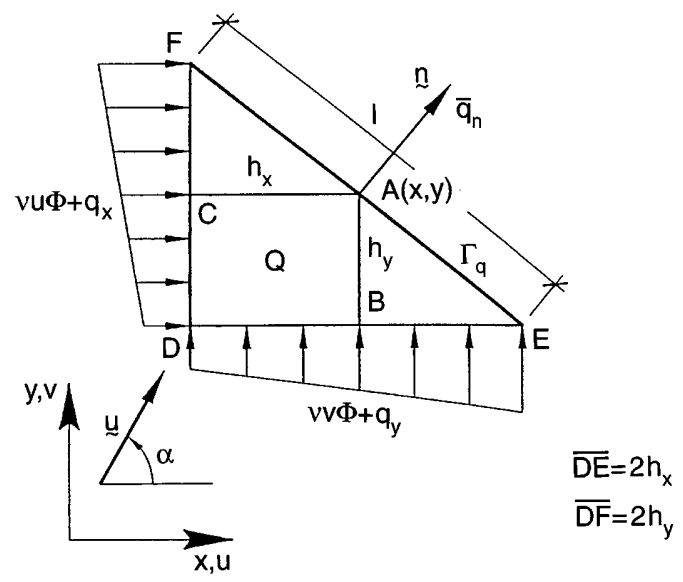

Fig. 3. Balance of fluxes in a two-dimensional domain next to a Neumann boundary $\mathbf{u}=[u, v]^{\mathrm{T}}$ forms with the global $x$ axis. The stabilized governing equations (27) and (34) can now be written as: Steady state

$r-\frac{h}{2|\mathbf{u}|} \mathbf{u}^{\mathrm{T}} \nabla r=0$

\section{Transient}

$c \frac{\partial \phi}{\partial t}-r-\frac{h}{2|\mathbf{u}|} \mathbf{u}^{\mathrm{T}} \nabla\left(c \frac{\partial \phi}{\partial t}-r\right)=0$.

In the derivation of above expressions, use of the identities $\sin \alpha=u /|\mathbf{u}|$ and $\cos \alpha=v /|\mathbf{u}|$ has been made.

The intrinsic time in $2 \mathrm{D}$ advective-diffusive problems is defined now as (Hughes and Mallet 1986)

$\tau=\frac{h}{2|\mathbf{u}|}$

With this definition Eqs. (35a,b) can be rewritten as:

Steady state

$r-\tau \mathbf{u}^{\mathrm{T}} \nabla r=0 \quad$ on $\Omega$

$(37 a)$

Transient

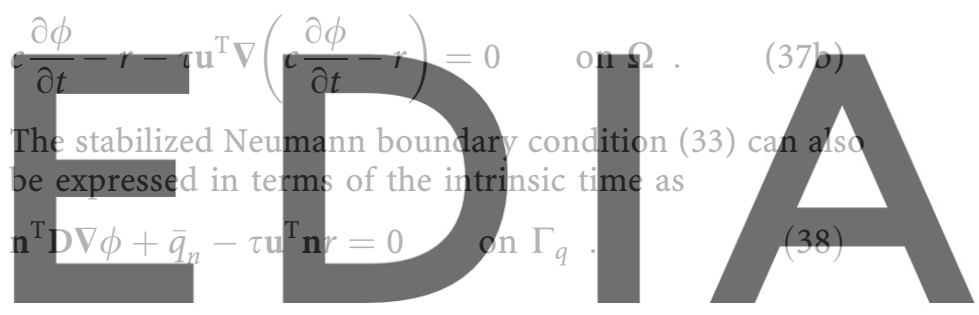

5.3

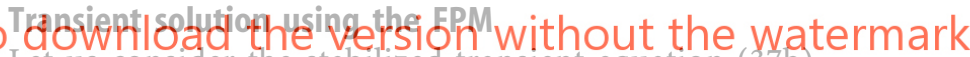
Let us consider the stabilized transient equation (37b) neglecting for simplicity the effect of the time derivative in the stabilization term. A simple explicit integration from $t^{n}$ to $t^{n+1}=t^{n}+\Delta t$ gives

$\Delta \phi=\frac{\Delta t}{c}\left[r-\tau \mathbf{u}^{\mathrm{T}} \nabla r\right]^{n}$.

Equation (39) can be found to be identical to that obtained using a characteristic approximation as described in Zienkiewicz and Codina (1995) and Zienkiewicz et al. (1995), if the intrinsic time is taken to coincide with $\Delta t / 2$. Indeed Eq. (39) provides a more meaningful explanation of the stabilization term within the brackets.

Equation (39) can now be discretized in space using the FPM by substituting the interpolation (17) for the unknown $\phi$. Choosing a point collocation procedure leads to an explicit system of equations from which the values of $\Delta \phi$ can be obtained and the solution advanced in time in the usual manner. Indeed the value of the time step increment $\Delta t$ in (39) must be adequately chosen to ensure stability of the time integration scheme. Local time stepping can be used if only the steady state solution is needed.

Note that for the full success of the numerical scheme the stabilized form of the Neuman boundary conditions (38) must be used (Oñate et al. 1996b).

The expression of the stabilized term arising in Eq. (39) can be simplified if a linear or quadratic approximation is used as the cubic derivatives of $\phi$ in the diffusive term are 
zero. A further simplification arises if the source term is constant and the stabilizing term is then simply given by $\tau \mathbf{u}^{\mathrm{T}}\left(\mathbf{u}^{\mathrm{T}} \nabla \phi\right)$.

Note that the balancing term may be interpreted now as an additional diffusion as typically occurs in the FE literature (Zienkiewicz and Taylor 1981).

The value of the optimal intrinsic time $\tau$ can be found by using the same arguments of standard upwinding and Petrov-Galerkin FE procedures, i.e. by searching exact nodal values for the simple $1 \mathrm{D}$ problem with $Q=0$. Application of this concept to the FPM gives (Oñate et al. 1995a,b, 1996a,b):

$\tau= \begin{cases}\frac{\alpha h}{4|\mathbf{u}|} & \text { for linear interpolations }(m=2), \\ \frac{\alpha h}{8|u|} & \text { for quadratic interpolations }(m=3)\end{cases}$

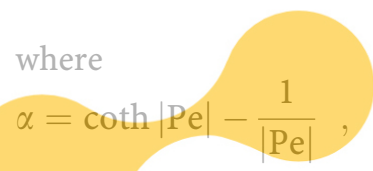

(41)

with the Peclet number defined as $\mathrm{Pe}=|\mathbf{u}| h / 2 k$.

In Eq. (42) $h$ is half the distance measured along the streamline between the end points for a particular cloud as shown in Fig. 4.

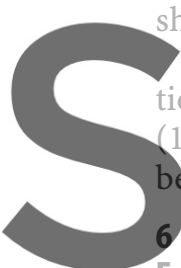

An alternative procedure for computing the stabilizaion parameter based on the

used.

\section{Extension of the FPM to fluid flow probtems}

\section{Registe ${ }^{6 . f o r}$ free at https//www.scipedia.com to}

The approach proposed in previous section will be generalized now to solve two dimensional compressible fluid mechanics problems governed by the Navier-Stokes equations written in conservation form as (Zienkiewicz and Taylor 1991)

$\mathbf{A}(\mathbf{v})=\frac{\partial \mathbf{v}}{\partial t}+\frac{\partial \mathbf{f}_{i}}{\partial x_{i}}+\frac{\partial \mathbf{g}_{i}}{\partial x_{i}}+\mathbf{q}=0 \quad i=1,2$,

where

$$
\begin{aligned}
\mathbf{v}= & {\left[\rho, \rho u_{1}, \rho u_{2}, \rho u_{3}, \rho E\right]^{\mathrm{T}} } \\
\mathbf{f}_{i}= & {\left[\rho u_{i}, \rho u_{1} u_{i}+\delta_{1 i} p, \rho u_{2} u_{i}\right.} \\
& \left.+\delta_{2 i} p, \rho u_{3} u_{i}+\delta_{3 i} p, u_{i}(\rho E+p)\right]^{\mathrm{T}}
\end{aligned}
$$

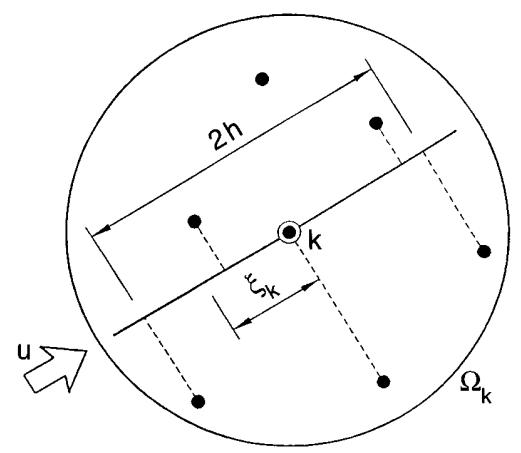

Fig. 4. Definition of the characteristic length $h$ defines the convective flux vector and

$\mathbf{g}_{i}=\left[0,-\tau_{1 i},-\tau_{2 i},-\tau_{3 i},-k \frac{\partial T}{\partial x_{i}}-\tau_{i j} u_{j}\right]^{\mathrm{T}}$

defines the diffusion flows. Finally

$\mathbf{q}=\left[0, g_{1}, g_{2}, g_{3}, 0\right]^{\mathrm{T}}$

gives the source terms due to (minus) the gravity acceleration. In above $\rho$ is the density, $u_{k}$ is the velocity in the $k$ th direction $p$ is the pressure and $E$ is the energy. The stress components $\tau_{i j}$ are related to velocity gradients by

$\tau_{i j}=\mu\left(\frac{\partial u_{i}}{\partial x_{j}}+\frac{\partial u_{j}}{\partial x_{i}}-\frac{2}{3} \frac{\partial u_{k}}{\partial x_{k}} \delta_{i j}\right)$,

where $\mu$ is the viscosity. The equations are completed by the universal gas law

$p=\rho R T$

where $R$ is the gas constant and $T$ the temperature.

6.2

Stabilization of compressible flow equations

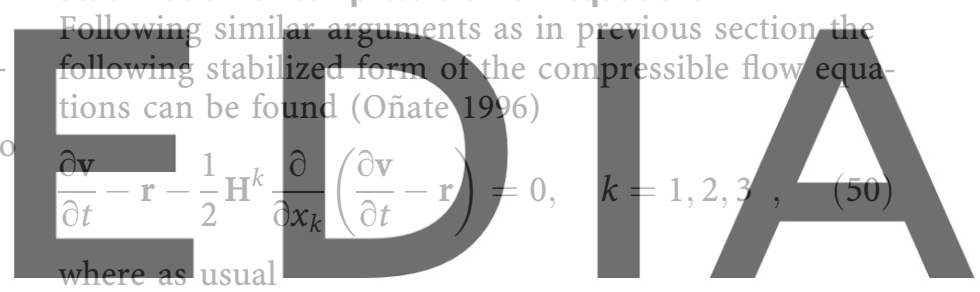

\section{$\partial \mathbf{f}_{i} \quad \partial \mathbf{g}_{i}$}

Matrix $\mathbb{H}^{k}$ appearing in the stabilizing term of Eq. (50) can be obtained as

$\mathbf{H}^{k}=\left[\begin{array}{ccccc}h_{\rho}^{k} & & & & 0 \\ & h_{u}^{k} & & & \\ & & h_{u}^{k} & & \\ & & & h_{u}^{k} & \\ 0 & & & & h_{e}^{k}\end{array}\right], \quad k=1,2,3$.

In above $h_{\rho}^{k}, h_{u}^{k}, h_{e}^{k}, k=1,2,3$ are the dimensions of the right prism where mass, momentum and energy balances are enforced, respectively. Note that for $\mathbf{H}^{k}=0$ the standard conservation form of the compressible flow equation is recovered.

\section{3}

Definition of characteristic lengths, intrinsic times and stabilized traction boundary conditions

The following characteristic lengths for each of the five equations governing mass, momentum and energy balances can be now defined as

$$
\begin{array}{lll}
h_{\rho}=\left|\mathbf{h}_{\rho}\right| \quad \text { with } & \mathbf{h}_{\rho}=\left[h_{\rho}^{1}, h_{\rho}^{2}, h_{\rho}^{3}\right]^{\mathrm{T}}, \\
h_{u}=\left|\mathbf{h}_{u}\right| \quad \text { with } & \mathbf{h}_{u}=\left[h_{u}^{1}, h_{u}^{2}, h_{u}^{3}\right]^{\mathrm{T}} \\
h_{e}=\left|\mathbf{h}_{e}\right| & \text { with } \quad \mathbf{h}_{e}=\left[h_{e}^{1}, h_{e}^{2}, h_{e}^{3}\right]^{\mathrm{T}}
\end{array}
$$


It will now be assumed that the characteristic length vec- with tors $\mathbf{h}_{\rho} \mathbf{h}_{u}$ and $\mathbf{h}_{e}$ have all the direction of the velocity vector $\mathbf{u}=\left[u_{1}, u_{2}, u_{3}\right]^{\mathrm{T}}$. This allows us to express matrix $\mathbf{H}^{k}$ as

$\mathbf{H}^{k}=\frac{u_{k}}{|\mathbf{u}|} \mathbf{H} \quad$ with $\quad \mathbf{H}=\left[\begin{array}{lllll}h_{\rho} & & & & 0 \\ & h_{u} & & & \\ & & h_{u} & & \\ & & & h_{u} & \\ 0 & & & & h_{e}\end{array}\right]$

and the stabilized equations have now the following form

$\frac{\partial \mathbf{v}}{\partial t}-\mathbf{r}-\frac{u_{k}}{2|\mathbf{u}|} \mathbf{H} \frac{\partial}{\partial x_{k}}\left(\frac{\partial \mathbf{v}}{\partial t}-\mathbf{r}\right)=0$.

The intrinsic time for each of the balancing equations can be defined as

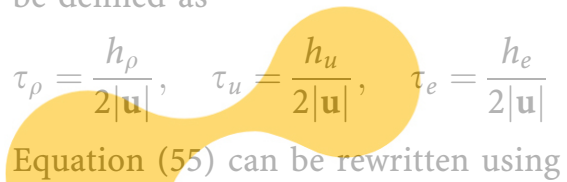

Equation (55) can be rewritten using (56) as

$\frac{\partial \mathbf{v}}{\partial t}-\mathbf{r}-[\tau] u_{k} \frac{\partial}{\partial x_{k}}\left(\frac{\partial \mathbf{v}}{\partial t}-\mathbf{r}\right)=0$
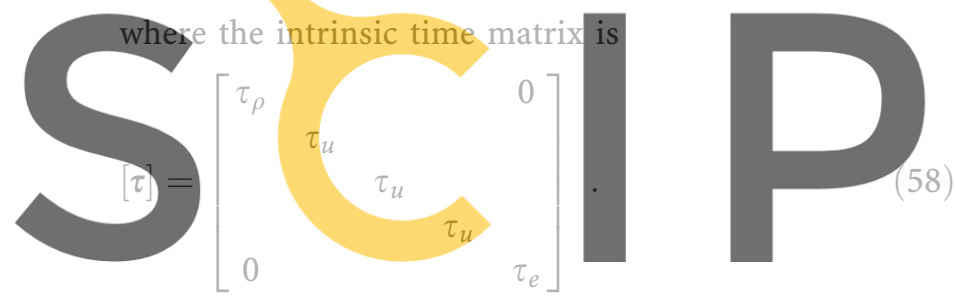

Regisimilar higher order balance concepts can be anplied to

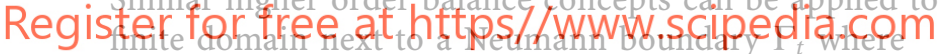

tractions $\overline{\mathrm{t}}$ are specified (see Fig. 5). The resulting stabi-

lized form of the traction boundary condition can be

written as (Oñate 1996, 1998)

$\mathbf{M} \boldsymbol{\sigma}-\overline{\mathbf{t}}-\frac{h_{u}}{2|\mathbf{u}|} \mathbf{n}^{\mathrm{T}} \mathbf{u r}_{m}=0$

where $\mathbf{n}$ is the normal vector

$\boldsymbol{\sigma}=\left[\sigma_{11}, \sigma_{22}, \sigma_{33}, \sigma_{12}, \sigma_{13}, \sigma_{23}\right]^{\mathrm{T}}$

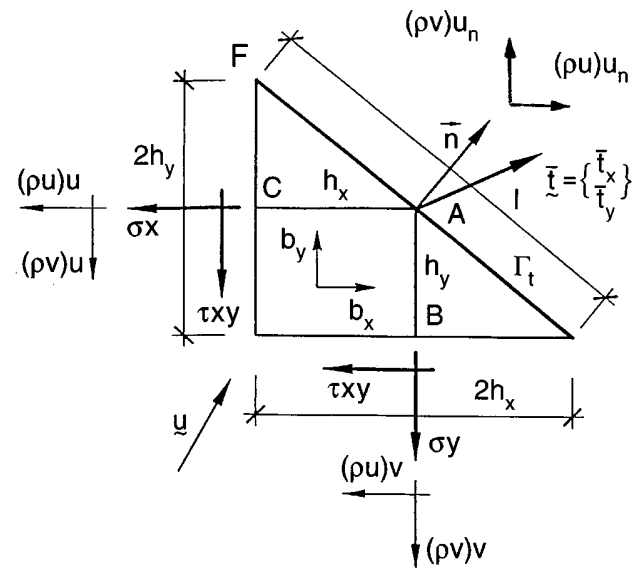

$\begin{aligned} \sigma_{i j} & =\tau_{i j}-p \delta_{i j} \\ \mathbf{M} & =\left[\begin{array}{cccccc}n_{x} & 0 & 0 & n_{y} & n_{z} & 0 \\ 0 & n_{y} & 0 & n_{x} & 0 & n_{z} \\ 0 & 0 & n_{z} & 0 & n_{x} & n_{y}\end{array}\right]\end{aligned}$

and $\mathbf{r}_{m}$ contains the momentum equations, i.e. the rows 2 , 3 , and 4 of vector $\mathbf{r}$ of Eq. (51).

Indeed for boundaries where the outgoing heat flux is specified the stabilized form given by Eq. (38) should be used.

The equivalent stabilized equations (57) are the basis for the numerical solution of compressible flow problems using the FPM. As usual the time integration can be performed prior to the application of the spatial discretization scheme. Neglecting the time derivative in the stabilization term and using a simple forward integration gives the following expression

$\Delta \mathbf{v}=\Delta t\left[\mathbf{r}-[\tau] u_{k} \frac{\partial \mathbf{r}}{\partial x_{k}}\right]^{n}$

where $\Delta \mathbf{v}=\mathbf{v}^{n+1}-\mathbf{v}^{n}$.

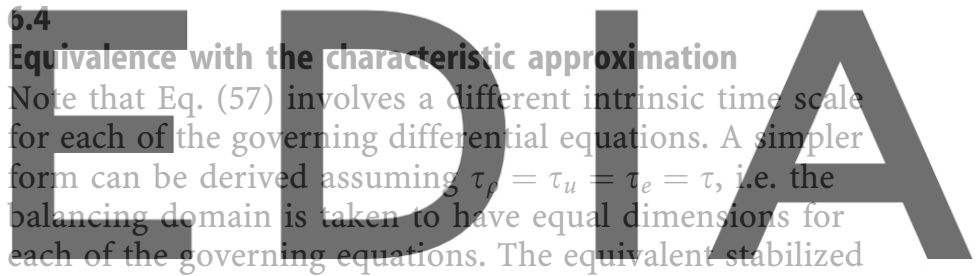

equations can be written as

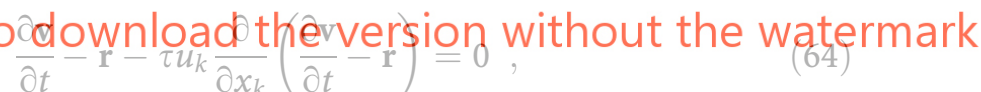

where

$h=|\mathbf{h}| \quad$ with $\quad \mathbf{h}=\left[h^{1}, h^{2}, h^{3}\right]^{\mathrm{T}}$,

$\overline{\mathbf{H}}^{k}=h^{k} \mathbf{I}_{5} \quad$ and $\quad \tau=\frac{h}{2|\mathbf{u}|}$,

where $I_{5}$ is the $5 \times 5$ unit matrix.

The explicit integration of Eq. (64) gives (neglecting the time derivative in the stabilization term)

$\Delta \mathbf{v}=\Delta t\left[\mathbf{r}-\tau u_{k} \frac{\partial \mathbf{r}}{\partial x_{k}}\right]^{n}$.

This expression is found again to be analogous as that derived via characteristic Galerkin procedures if the intrinsic time is taken to be equal to $\Delta t / 2$ (Zienkiewicz and Codina 1995; Zienkiewicz et al. 1995).

Equation (66) can now be taken as the basis for the spatial discretization using the FPM. The procedure follows precisely the same steps as described in a previous section for advective-diffusive problems.

6.5

\section{Stabilized equations for incompressible flows}

Assuming the flow to be isothermal and the density to be

Fig. 5. Flow domain next to a Neumann boundary where surface tractions are prescribed the incompressible low equations: 
Continuity equation

$\frac{\partial u_{i}}{\partial x_{i}}-\frac{h_{p}^{k}}{2} \frac{\partial}{\partial x_{k}}\left(\frac{\partial u_{i}}{\partial x_{i}}\right)=0 ;$

Momentum equations

$\frac{\partial \mathbf{u}}{\partial t}-\mathbf{r}_{m}-\frac{1}{2} \mathbf{H}_{m}^{k} \frac{\partial}{\partial x_{k}}\left(\frac{\partial \mathbf{u}}{\partial t}-\mathbf{r}_{m}\right)=0$

where

$$
\begin{aligned}
& \mathbf{u}=\left[u_{1}, u_{2}, u_{3}\right]^{\mathrm{T}}, \quad \mathbf{H}_{m}^{k}=h_{u}^{k} \mathbf{I}_{3} ; \\
& \mathbf{r}_{m}=-\left[\nabla \mathbf{u}^{\mathrm{T}}\right] \mathbf{u}-\frac{1}{\rho} \boldsymbol{\nabla} p+\frac{1}{\rho} \mathbf{L}^{\mathrm{T}} \boldsymbol{\sigma}+\mathbf{b},
\end{aligned}
$$

with

$$
\begin{aligned}
& \mathbf{b}=\left[f_{1}, f_{2}, f_{3}\right]^{\mathrm{T}}, \boldsymbol{\nabla}=\left[\frac{\partial}{\partial x}, \frac{\partial}{\partial y}, \frac{\partial}{\partial z}\right]^{\mathrm{T}} ; \\
& \mathbf{L}^{\mathrm{T}}=\left[\begin{array}{cccccc}
\partial / \partial x & 0 & 0 & \partial / \partial y & \partial / \partial z & 0 \\
0 & \partial / \partial y & 0 & \partial / \partial x & 0 & \partial / \partial z \\
0 & 0 & \partial / \partial z & 0 & \partial / \partial x & \partial / \partial y
\end{array}\right]
\end{aligned}
$$

Fig. 6. Grid of 14106 points for the solution of viscous flow around a NACA0012 airfoil $(n=5, m=3)$

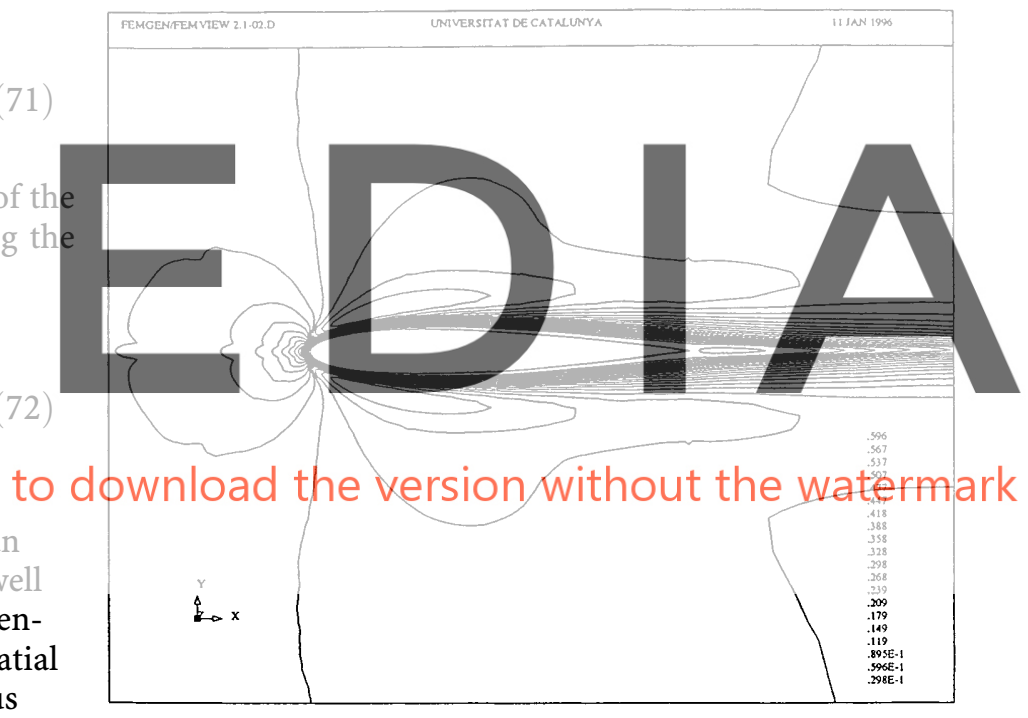

Fig. 7. Mach number contours for the solution of viscous flow around a NACA0012 airfoil $\left(\operatorname{Re}=5000, M_{\infty}=0.5, \alpha=0^{\circ}\right)$ using 141106 points

Mavripilis et al. (1989). Figure 11 shows the pressure contours on the wake region showing the expected parallel distribution of lines and Fig. 12 shows an acceptable rate of convergence of the results.

\section{8}

\section{Conclusions}

In the paper details of the derivation of a stabilized finite point method (FPM) for analysis of advective-diffusive transport and compressible/incompressible fluid flow problems have been presented. Recent applications of the FPM indicate that it is a promising "meshless" numerical procedure for analysis of a wide range of CFD problems. Further testing is in progress, including the necessary extension of solving three dimensional problems where the efficiency of the FPM should become more apparent. 


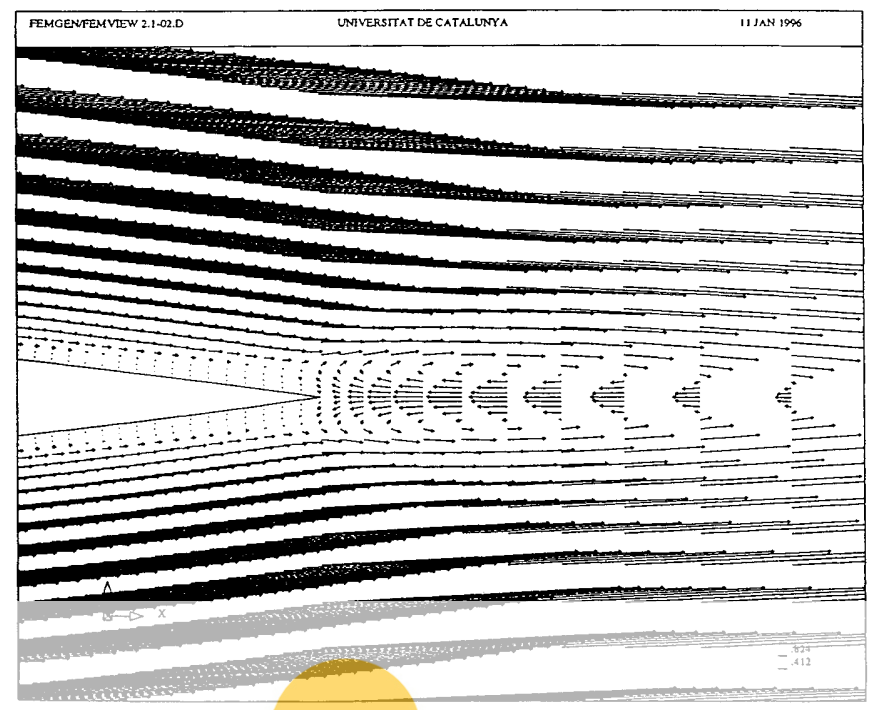

Fig. 8. Velocity vectors at the trailing edge for the solution of viscous flow around a NACA0012 airfoil. Note the recirculation at tip of the trailing edge

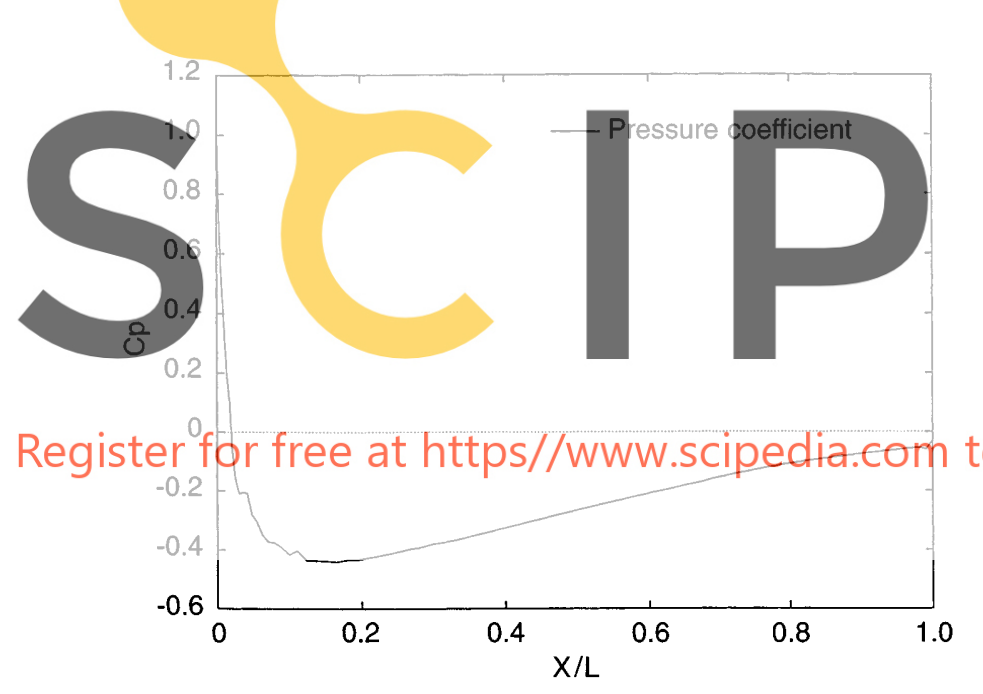

Fig. 9. Pressure coefficient along the airfoil for the solution of viscous flow around a NACA0012 airfoil

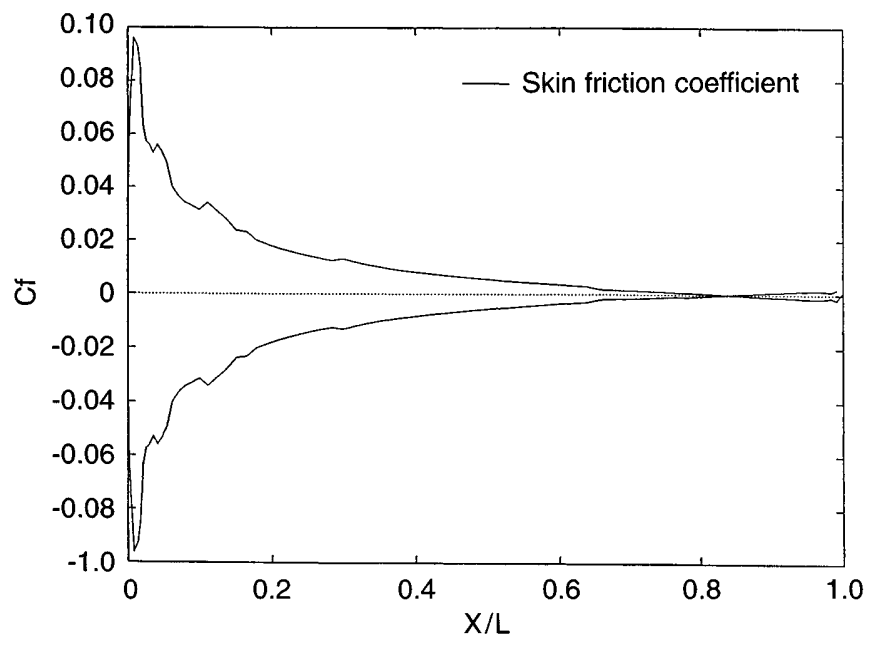

Fig. 10. Skin friction coefficient along the airfoil for the solution of viscous flow around a NACA0012 airfoil

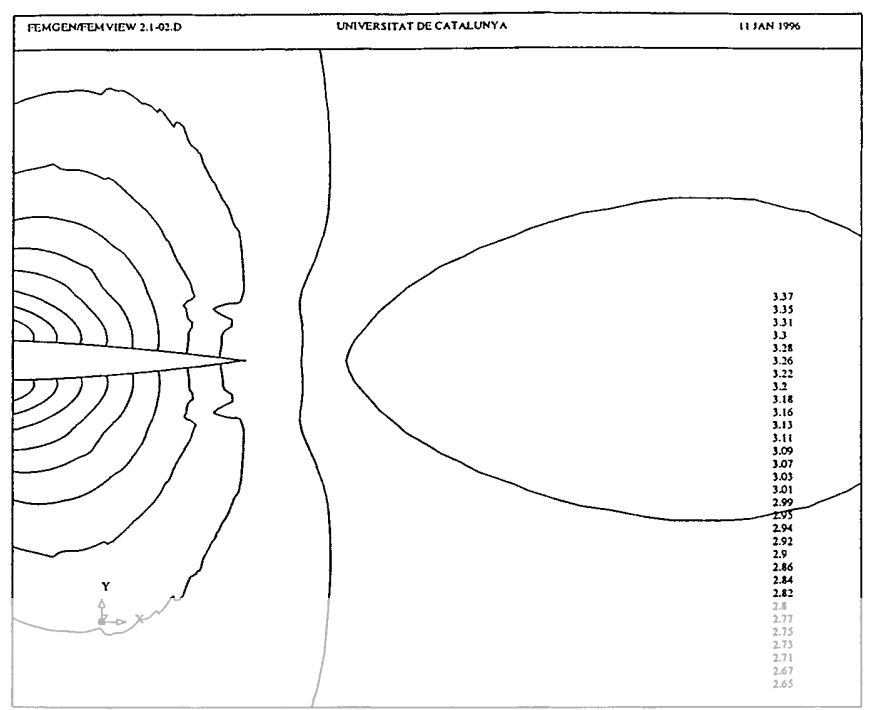

291

Fig. 11. Pressure lines in the wake for the solution of viscous flow around a NACA0012 airfoil. Note their parallel position to each other in the wake

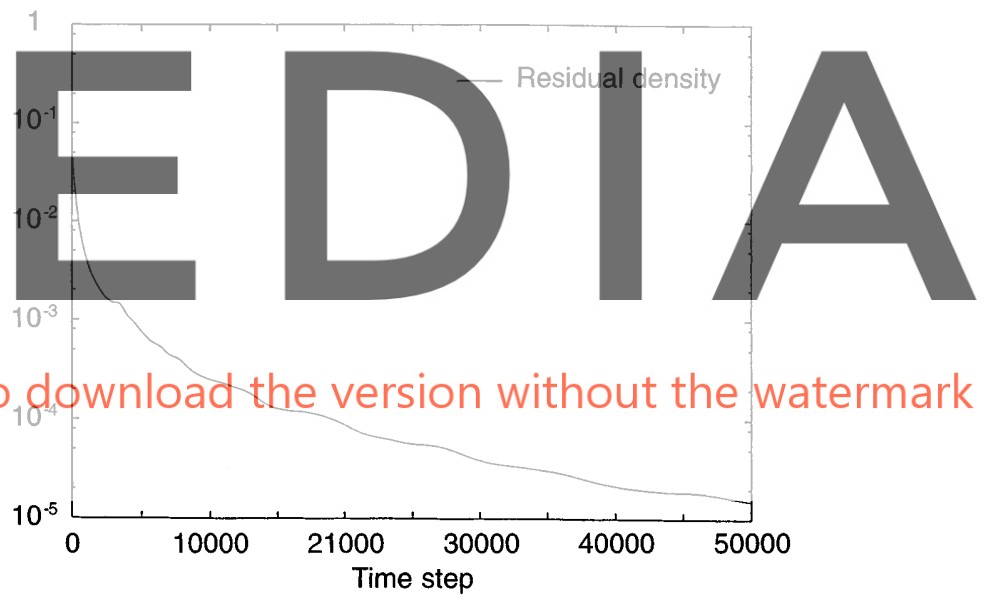

Fig. 12. Convergence history of the density residual for the solution of viscous flow around a NACA0012 airfoil $(\mathrm{Re}=5000$, $M_{\infty}=0.5, \alpha=0^{\circ}$ )

\section{References}

Babuška I, Melenk JM (1995) The partition of unity finite element method. Technical Note EN-1185, Institute for Physical Science and Technology. Univ. Maryland

Batina J (1993) A Gridless Euler/Navier-Stokes solution algorithm for complex aircraft applications. AIAA 93-0333, Reno NV, 11-14 Jan

Belytschko T, Lu Y, Gu L (1994) Element free Galerkin methods. Int. J. Num. Meth. Eng. 37:229-56

Belytschko T, Organ D, Krongauz Y (1995) A coupled finiteelement-element-free Galerkin Method. Comput. Mech. 17:186195

Dolbow J, Belytschko T (1998) An introduction to programming the meshless element free Galerkin Method. In: Arch. in Comp. Meth. in Eng. (in print)

Duarte CA, Oden JT (1995) $H_{p}$ clouds - A meshless method to solve boundary-value problems. TICAM Report 95-05

Fischer T (1996) A contribution to adaptive numerical solution of compressible flow problems. Ph.D. Thesis, Universidad Politécnica de Cataluña, Monogr. No. 34, CIMNE, Barcelona 
Fisher T, Idelsohn I, Oñate E (1995) A meshless method for analysis of high speed flows. AGARD Meeting, Seville Forsythe GE, Wasow WR (1960) Finite Difference Methods for Partial Differential Equations. Wiley, New York

Hirsch C (1990) Numerical computations of internal and external flow, Vol. 2. Wiley, New York

Hughes TJR, Mallet M (1986) A new finite element formulation for computational fluid dynamics. III: The generalized streamline operator for multidimensional advective-diffusive systems.

Comp. Meth. in Appl. Mech. and Eng. 58:305-28

Idelsohn S, Oñate E (1994) Finite element and finite volumes.

Two good friends. Int. J. Num. Meth. Eng. 37:3323-3341

Liszka T (1984) An interpolation method for an irregular set of nodes. Int. J. Num. Meth. Eng. 20:1594-1612

Liszka T, Orkisz J (1990) The finite difference method at arbitrary irregular grids and its application in applied mechanics. Comput. Struct. 11:83-95

Liu WK, Chen Y (1995) Wavelet and multiple scale reproducing Kernel methods. Int. J. Num. Meth. Fluids 21:901-933

Liu WK, Jun S, Zhang YF (1995a) Reproducing Kernel particle methods. Int. J. Num. Meth. Fluids 20:1081-1106

Liu WK, Jun S, Li S, Adee J, Belytschko T (1995b) Reproducing Kernel particle methods for structural dynamics. Int. J. Num. Meth. Eng. 38:1655-1679

Liu WK, Chen Y, Chang CT, Belytschku T (1996a) Advances in Multiple Scale Kernel Particle Methods. Comput. Mech. 18:73111

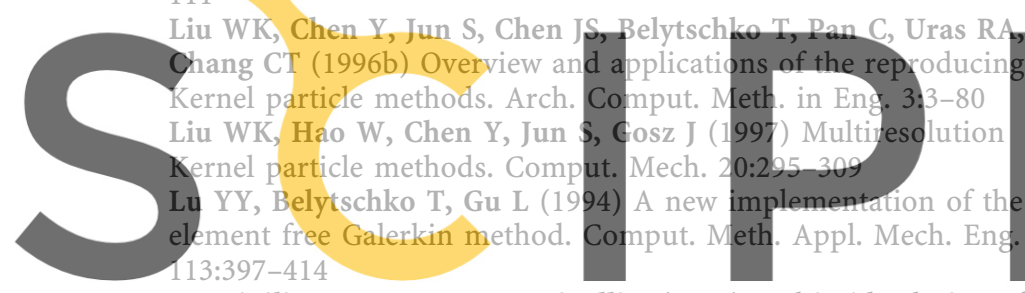

Mavripilis P, Jameson A, Matinelli L (1989) Multigrid solution of the Navier-Stokes equations on triangular meshes. ICASE Report

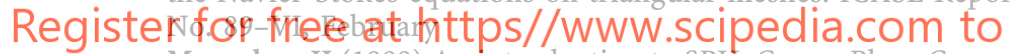
Moraghan JJ (1988) An introduction to SPH. Comp. Phys. Comm. 48:89-96

Nayroles B, Touzot G, Villon P (1992) Generalizing the FEM: Diffuse approximation and diffuse elements. Comput. Mech. 10, 307-18

Oñate E (1996) On the stabilization of numerical solution of convective transport and fluid flow problems. Research Report 81 CIMNE, Barcelona, Feb
Oñate E (1998) Derivation of stabilized equations for numerical solution of advective-diffusive Transport and fluid flow problems. Comp. Meth. Appl. Mech. Eng., 151:233-265

Onate E, Cervera M, Zienkiewicz OC (1994) A finite volume

format for structural mechanics. Int. J. Num. Meth. Eng. 37:181201

Oñate E, Idelsohn S, Zienkiewicz OC (1995a) Finite point methods in computational mechanics. Research Report 67, CIMNE, Barcelona, July

Oñate E, Idelsohn S, Zienkiewicz OC, Fisher T (1995b) A finite point method for analysis of fluid flow problems. Proc. 9th Int. Conf. on Finite Element Methods in fluids. Venize, Italy, 15-21 Oct

Oñate E, Idelsohn S, Zienkiewicz OC, Taylor RL (1996a) A finite point method in computational mechanics. Applications to convective transport and fluid flow. Int. J. Num. Meth. Eng. 39:38393866

Oñate E, Idelsohn S, Zienkiewicz OC, Taylor RL (1996b) A stabilized finite point method for analysis of fluid mechanics's problems. Comp. Meth. in Appl. Eng. 139:315-347

Oñate E, Garcia J, Idelsohn S (1997) Computation of the stabilization parameter for the finite element solution of advectivediffusive problems. Int. J. Num. Meth. Fluids, 25:1385-1407 Peraire J, Peiro J, Formaggia L, Morgan K, Zienkiewicz OC (1988) Finite element Euler computations in three dimensions. Int. J. Num. Meth. Eng. 26:2135-59

Perrone N, Kao R (1975) A general finite difference method for

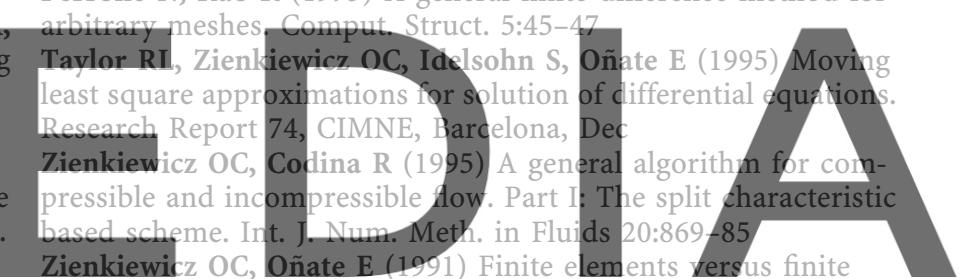

volumes. Is there a choice?. In: Wriggers P, Wagner W (eds) Non Linear Computational Mechanics. State of the Art, Springer,

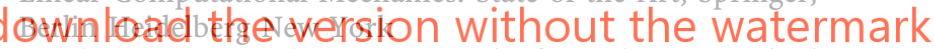

Zienkiewicz OC, Taylor RL (1991) The finite element method. Mc Graw Hill, New York

Zienkiewicz OC, Morgan K, Satya Sai BVK, Codina R, Vazquez M (1995) A general algorithm for compressible and incompressible flow. Part II: Tests on the explicit form. Int. J. Num. Meth. in Fluids 20:886-913 